\title{
Vital Signs Time Point Reference
}

National Cancer Institute

\section{Source}

National Cancer Institute. Vital Signs Time Point Reference. NCI Thesaurus. Code C83468.

The point in time that acts as a fixed reference point to a vital signs assessment. 